„This is the peer reviewed version of the following article: Bin Cai, Arezoo Dianat, René Hübner, Wei Liu, Dan Wen, Albrecht Benad, Luisa Sonntag, Thomas Gemming, Gianaurelio Cuniberti and Alexander Eychmüller (2017). Multimetallic Hierarchical Aerogels: Shape-engineering of the Building Blocks for efficient electrocatalysis. Advanced Materials, 2017, Volume 29, Issue 11, pp. 1605254, which has been published in final form at DOI:10.1002/adma.201605254.

This article may be used for non-commercial purposes in accordance with Wiley Terms and Conditions for Self-Archiving."

\title{
Multimetallic Hierarchical Aerogels: Shape-engineering of the Building Blocks for efficient electrocatalysis
}

Bin Cai, Arezoo Dianat, René Hübner, Wei Liu, Dan Wen, Albrecht Benad, Luisa Sonntag, Thomas Gemming, Gianaurelio Cuniberti and Alexander Eychmüller*

Bin Cai, Dr. Wei Liu, Dr. Dan Wen, Albrecht Benad, Luisa Sonntag, Prof. Dr. Alexander Eychmüller

Physikalische Chemie, Center for Advancing Electronics Dresden

Technische Universität Dresden

Bergstraße 66b, 01062 Dresden, Germany

E-mail: alexander.eychmueller@chemie.tu-dresden.de

Dr. Arezoo Dianat, Prof. Dr. Gianaurelio Cuniberti

Institute for Materials Science and Max Bergmann Center of Biomaterials

Center for Advancing Electronics Dresden

Technische Universität Dresden

Hallwachsstraße 3, 01069 Dresden, Germany

Dr. René Hübner

Helmholtz-Zentrum Dresden-Rossendorf

Bautzner Landstraße 400, 01328 Dresden, Germany

Dr. Thomas Gemming

Institute for Complex Materials, IFW Dresden

P.O. Box, D-01171 Dresden, Germany

Keywords: aerogels, hierarchical structures, sol-gel processes, electrocatalysts, ethanol oxidation

Construction of macroscopic materials and devices from nanocrystals (NCs) with desirable physical and chemical properties has become one of the holy grails of the booming nanotechnology due to its essential role in fundamental research and commercial relevance. ${ }^{[1]}$ Among them, aerogels assembled from colloidal NCs are of enormous scientific and technological interest owing to their ultralow density, high surface area and large open 
interconnected pores. ${ }^{[2-4]}$ Over the past decade, various kinds of inorganic nano building blocks (NBBs), varying from semiconductor NCs, metal oxides, metal NCs, etc., have been employed to design aerogel monoliths and explore their improved properties, in areas such as photovoltaics, energy storage, and catalysis. ${ }^{[5-9]}$ The aerogels built from the NBBs can inherit the properties and functions from the parent NBBs while maintaining the aerogel properties, which frequently leads to amplification of the inherited properties and results in features that are unique to the aerogels. ${ }^{[10]}$ For instance, pure metallic aerogels are promising electrocatalysts due to the intrinsic advantages from both metal NCs and aerogels, including metallic backbone (enables rapid electron transfer), large surface area (provides more reactive sites), high porosity (accelerates mass transfer), and self-supportability (eliminates support corrosion). ${ }^{[11]}$ To date, improved electrocatalytic activities have been demonstrated on metallic aerogels derived from noble metals (e.g. Pd, Pt, Au) ${ }^{[12-15]}$ Nonetheless, the investigation of aerogel electrocatalysts is still in the early stages and is largely limited by the solid NBBs and simplified compositions. ${ }^{[11]}$ The previous reports have demonstrated that the intimate relationship between the NBBs and the resulting aerogels offers opportunities to optimize the aerogel properties by tailoring the NBBs. ${ }^{[16]}$ Thus, it holds great promise to endow aerogel electrocatalysts with improved catalytic properties by optimizing the metal NBBs in terms of morphology and alloying.

As the most basic units of metallic aerogels, namely the metal NCs have been extensively investigated based on the surfactant-assisted precision synthesis that provides monodispersity, favourable morphologies, and controlled surface properties. ${ }^{[17]}$ For instance, galvanic replacement offers a facile and versatile route to a variety of advanced NCs that often characterized by hollow interiors and tunable compositions. ${ }^{[18]}$ Nevertheless, the typical sol-gel synthesis for aerogels is based on the controlled destabilization of the NC sols, which is 
generally achieved by slight depleting of stabilization or partial removal of the surfactants. ${ }^{[19]}$ In this respect, the demand of surfactant throughout the synthesis obstructs the destabilizationgelation step, rendering them stagnant in crossing the "sol-gel" bridge from nano materials to macro aerogels. Therefore, despite the tremendous advancements in nanotechnology, the development of aerogels via the regulation of the NBBs remains one of the greatest challenges. In particular, the research of electrocatalysis on metallic aerogels lags far behind the flourishing nanoscience. From the perspective of noble-metal-based catalysts, tailoring the morphology and transition metal doping remain the most efficient ways to improve their catalytic performance. However, these concepts remain quite difficult to be realized in the design of aerogel catalysts due to the incompatible reduction kinetics of multiple metal precursors and the hardlyimplemented destabilization-gelation process. Besides, although extensive efforts have been devoted to the galvanic synthesis, the underlying structural growth mechanism remains elusive to date and is highly desirable.

In this study, we firstly address these challenges by demonstrating a new class of hierarchical aerogels composed of multimetallic $\mathrm{Ni}-\mathrm{Pd}_{\mathrm{x}} \mathrm{Pt}_{\mathrm{y}} \mathrm{NBBs}$ with continuously engineered shape and compositions. Several important features can be highlighted: (1) the morphology of the NBBs was in situ engineered from hollow nanospheres (HNSs) to dendritic nanocrystals (DNCs), which were further applied to construct aerogel monoliths by a facile and large-scalable sol-gel method. This results in aerogels with hierarchical structures organizing the nanoscale regulated architecture and macroscale three-dimensional (3D) network structure, leading to an abundance of exposed edges and a high surface area (varying from 95.4 to $67.7 \mathrm{~m}^{2} \mathrm{~g}^{1}$ for different compositions). (2) The shape engineering was based on the surprisingly different behavior of $\mathrm{Pd}^{2+}$ and $\mathrm{Pt}^{2+}$ ions on galvanic replacement reactions with Ni NCs. With the aid of time- 
dependent transmission electron microscopy (TEM) and first principle molecular dynamics (MD) simulations, the structural growth mechanism underlying the galvanic replacement was revealed in terms of nanowelding of the particulate reaction intermediates. (3) These hierarchical aerogels combine the advantages from the joint hollow-dendritic morphologies, the multimetallic alloying and the aerogel structures, serving as significant roles in enhancing catalysis. As a demonstration, the $\mathrm{Ni}-\mathrm{Pd}_{60} \mathrm{Pt}_{40}$ aerogel exhibits remarkable electrocatalytic activity which is 10.6 and 7.6-fold higher than the state-of-the-art $\mathrm{Pd} / \mathrm{C}$ and Pt/C catalysts, respectively, taking ethanol oxidation reaction (EOR) as the example. Moreover, the durability of the hierarchical aerogels was largely improved due to the avoidance of carbon corrosion. Consequently, fundamental challenge to attain hierarchical aerogels with engineered NBBs is successfully addressed and these findings hold the potential for inspiring further development of galvanic synthesis.

The synthesis procedure of the multimetallic hierarchical $\mathrm{Ni}-\mathrm{Pd}_{\mathrm{x}} \mathrm{Pt}_{\mathrm{y}}$ aerogels is illustrated in Figure 1a. First, the Ni-Pdx $\mathrm{Pt}_{\mathrm{y}} \mathrm{NBBs}$ with controlled morphology and compositions were synthesized via a galvanic replacement method using Ni NCs as sacrificial templates under inert atmosphere (Figure S1). The Pd:Pt molar ratio can be precisely tuned by controlling of the precursor ratios, while fixing the Ni content at $\sim 20 \%$ (Figure S2). Since aqueous sols of the NBBs are usually very stable in the as-prepared state, it is necessary to concentrate the sols and eliminate most of the stabilizers to reach a "metastable state" (Without destabilization, the concentrated sols were stable for more than 1 month). Then, the hydrogel was achieved by heating the concentrated sols at $348 \mathrm{~K}$ for $6 \mathrm{~h}$ followed by ageing for 2 days. During the gelation, the NBBs connected and fused into a well-defined 3D necklace-like network structures. After the interstitial solvent of the resulting hydrogel was exchanged with acetone followed by supercritical drying, self-supported metallic aerogel monoliths were obtained (Figure S3). It 
should be noted that metallic aerogels are always fragile and will break into small fragments when bent or poked. High-resolution transmission electron microscopy (HR-TEM) images (Figure 1b-d and $\mathbf{h}-\mathbf{j}$ ) show the morphological evolution of the $\mathrm{Ni}^{-} \mathrm{Pd}_{\mathrm{x}} \mathrm{Pt}_{\mathrm{y}} \mathrm{NBBs}$ with continuously controlled compositions. The edge compositions exhibit entirely different structural behavior. The Pt free Ni-Pd NBBs exhibit a hollow-spherical shape with a diameter of $c a .23 \mathrm{~nm}$ and a shell thickness of $c a .3 \mathrm{~nm}$, whilst the Pd-free Ni-Pt NBBs show a dendritic morphology with sharp corners and edges. With increasing Pt ratio, the Pd-rich Ni-Pd $\mathrm{Pt}_{\mathrm{y}} \mathrm{NBBs}$ first show broken holes on the spherical shell and then gradually collapse. Eventually, the fragments connect to a branched structure until the overwhelming Pt ratio turns them into a dendritic morphology. Lattice fringes observed throughout the individual NBBs (Figure 1b-d and h-j) and the fused connection areas (Figure S4) reveal that the $\mathrm{Ni}^{-} \mathrm{Pd}_{\mathrm{x}} \mathrm{Pt}_{\mathrm{y}}$ aerogels are polycrystalline. Lattice spacings of about $2.31 \AA$, which can be indexed with the $\{111\}$ planes of a face-centeredcubic (fcc) structure, can be coherently extended across all locations. As shown in Figure 1e-g and k-m and Figure S5, all the Ni-Pdx $\mathrm{Pt}_{\mathrm{y}}$ aerogels have an extensive 3D network structure arising from random interconnection and fusion of individual NBBs during the gelation process. As shown in Figure 2a, X-ray diffraction (XRD) analysis further confirms the crystalline properties of the multimetallic aerogels. The typical diffraction peaks of fcc Pd/Pt shift to larger diffraction angles, indicating a lattice contraction induced by Ni alloying. Hence, the slight shrinkage of the lattice parameters can be explained by the permeation of $\mathrm{Ni}$ atoms into the $\mathrm{Pd} / \mathrm{Pt}$ lattice which results in smaller crystallographic unit cells. ${ }^{[16]}$ Scanning electron microscopy (SEM) images of the hierarchical aerogels (Figure 2b and S6) exhibit a high porosity and similarly interconnected networks for different compositions. The density was estimated to be 0.035-0.050 $\mathrm{g} \mathrm{cm}^{-3}$ (Figure S3), which is by far lower than the corresponding bulk metals. The 
specific surface area and porosity were further evaluated based on the $\mathrm{N}_{2}$ physisorption isotherms (Figure 2c and S7). The adsorption isotherm combines the characteristics of type II and type IV isotherms, indicating the wide spread of both meso- and macro-pores within the aerogel structures. As estimated from Brunauer-Emmett-Teller (BET) plots, the specific surface area of the Ni-Pdx $\mathrm{Pt}_{\mathrm{y}}$ aerogels ranges from 95.4 to $67.7 \mathrm{~m}^{2} \mathrm{~g}^{1}$, much higher than the values reported for other porous metals. ${ }^{[20]}$ Taking the Ni- $\mathrm{Pd}_{60} \mathrm{Pt}_{40}$ aerogel as an example (Figure 2c), the pore size distribution analysis exhibits several peaks at $c a .3-7 \mathrm{~nm}$ which can be attributed to the collapsed hollow cavity of the NBBs. Alloying of the aerogel structures was further confirmed by the elemental mapping based on energy-dispersive $\mathrm{X}$-ray spectroscopy in the scanning TEM (STEM) mode, where the Ni, Pd and Pt are distributed throughout the necklacelike backbones (Figure 2d and S8). X-ray photoelectron spectroscopy (XPS) was employed to investigate the surface properties of the aerogels (Figure 2e). The multimetallic aerogels exhibit higher ratio of metallic state $\left(\mathrm{Pd}^{0}\right.$ and $\left.\mathrm{Pt}^{0}\right)$ over oxide state $\left(\mathrm{Pd}^{\mathrm{II}}\right.$ and $\left.\mathrm{Pt}^{\mathrm{II}}\right)$, which may contribute to improved catalytic properties for electrocatalysis. ${ }^{[21]}$

Galvanic replacement reactions, accompanied with the Kirkendall effect, have been widely applied in the synthesis of hollow structures. ${ }^{[18]}$ However, in this work, it was employed to regulate the morphology from hollow-spherical to dendritic by tuning the oxidative elements. Given that the platinum-group metals have similar physiochemical properties (e.g. higher redox potentials and similar atomic radius), it is of significant interest to study the driving force that governs the composition-dependent morphologies. ${ }^{[22]}$ Herein, the most representative compositions (Ni-Pd and $\mathrm{Ni}-\mathrm{Pt}$ systems) are selected to study their structural growing mechanism. As shown in Figure 3a-c, we monitored the galvanic replacement at different reaction times by TEM to reveal the growth pathway of the Ni-Pd and Ni-Pt NBBs. Figure 3d 
schematically illustrates the major steps involved in the growth pathway deduced from the TEM images. Before the injection of Pd/Pt precursors, the Ni NPs show a spherical morphology (Figure 3a). After the injection of $\mathrm{Pd}^{2+} / \mathrm{Pt}^{2+}$ precursors, the galvanic reactions (Equation 1) on the Ni NPs are initiated immediately at the site with highest surface energy. ${ }^{[23]}$

$$
\mathrm{Ni}+\mathrm{PdCl}_{4}^{2-} \rightarrow \mathrm{Pd}+\mathrm{Ni}^{2+}+4 \mathrm{Cl}^{-} \& \mathrm{Ni}+\mathrm{PtCl}_{4}{ }^{2-} \rightarrow \mathrm{Pt}+\mathrm{Ni}^{2+}+4 \mathrm{Cl}^{-}
$$

As a result, $\mathrm{Ni}$ atoms are oxidized and released into the solution, whilst the electrons are captured by $\mathrm{Pd}^{2+} / \mathrm{Pt}^{2+}$ ions to generate Pd/Pt atoms. The TEM images (10s in Figure 3b-c and step 1 in Figure 3d) indicate that the newly formed $\mathrm{Pd} / \mathrm{Pt}$ atoms tend to nucleate as isolated intermediate nanoparticles (INPs) instead of forming a thin layer on the Ni surface. This thermodynamically driven behavior was also confirmed by MD simulations, as the nucleated atoms exhibit lower energy than the dispersed configuration (Figure S9). The nucleation of the INPs is always accompanied with alloying with template metals, rendering the INPs composed of Ni-Pd or NiPt. ${ }^{[18]}$ As the reaction proceeded (30s in Figure 3b-c and step 2 in Figure 3d), the INPs with a diameter of $\sim 2 \mathrm{~nm}$ gradually cover the surface and the gaps between them will allow the $\mathrm{Pd}^{2+} / \mathrm{Pt}^{2+}$ ions to diffuse in and $\mathrm{Ni}^{2+}$ to diffuse out of the cavity. During and after the deposition of the INPs, the dissolution of the Ni interior (after 30s in Figure 3b-c and step 2 in Figure 3d) is ascribed to not only the galvanic consumption, but also the instability of $\mathrm{Ni}$ in the presence of Pd and Pt (Figure S10-11).

The growth pathway of the Ni-Pd and Ni-Pt systems are similar in the early steps of the galvanic reaction, both leading to the nucleation of alloyed INPs on the surface. However, as shown in Figure 3b-c (1 min) and $\mathbf{d}$ (step 3), differences start to appear after a large portion of the $\mathrm{Ni}$ interior is dissolved. During the dissolution of the Ni interior, the surface INPs gradually lose their support and easily collapse. However, if the surface INPs can connect and weld into a self- 
supported shell structure before full dissolution of the inner Ni atoms, such collapse can be prevented. Therefore, we propose a growth mechanism that ascribes the morphological differences to the "nanowelding ability" of the surface NiPd or NiPt INPs (Scheme S1a).

Welding of nanoscale metals is a solid-state process that occurs by transport of atoms via atomic dissolution and surface relaxation to reduce the surface free energy. ${ }^{[24,25]}$ To verify the welding difference of the NiPd and NiPt INPs, MD simulations at $600 \mathrm{~K}$ were carried out to track the welding process of two contacted particles in the form of $\mathrm{Ni}_{20} \mathrm{Pd}_{80}$ and $\mathrm{Ni}_{20} \mathrm{Pt}_{80}$ (the compositions are set according to the final aerogels). The alloyed NiPd and NiPt particles are set with polyhedral shape and fcc structure bound mainly with (111) facets. As shown in Figure 4a and Movie S1-2, the NiPd particles with fcc structure came into contact with a contact area limited to 2 atoms. Then, atomic diffusion on the metallic surface was thermodynamically activated and the surface atoms seem more energetic than those in the interior. After 7ps, the contact area is increased due to interparticle diffusion of atoms, indicating the welding of the two particles. After 15 ps, the integration of two particles proceeds further. The NiPt particles display a similar welding behavior. To differentiate the welding ability of the two systems, we calculated the total surface area as a function of time as shown in Figure $\mathbf{4 b}$. Since the degree of integration of two particles is proportional to the decrease of their total surface area, it is clearly seen that the NiPd particles shows better welding ability, which indicates a faster fusion and connection of the particles. At the same time, NiPt particles need more time to fully fuse and get connected.

The differences observed in the welding simulation of the NiPd and NiPt particles agree well with the experimental results. As the Pd/Pt atoms deposit and nucleate on the Ni surface, the $\mathrm{Ni}$ interior continues to dissolve which makes the surface pre-formed NiPd and NiPt INPs becoming gradually unsupported (Step a in Scheme S1a). Due to the incomplete separation between the 
INPs and Ni support, the surface INPs will fall towards the center during the gradually depriving of the interior support. During the surface INPs "walking" into the center, the Brownian motion may let them collide for a certain probability. Because of the better welding ability, the NiPd INPs are easier to fuse and connect once they touch each other. Thus the dissolution of the inner Ni support results in a smooth hollow shell structure. However, the welding and connection of NiPt INPs is insufficient during the relatively fast losing of Ni support, thus results in a collapse of the partially-connected surface INPs and then the formation of a dendritic structure. It should be noted that the fusion between the NBBs during the followed gelation process is different from the nanowelding behavior of the INPs (Scheme S1b).

It is reasonable to anticipate that the hierarchical aerogels are electrochemically more accessible due to the combination of a regulated architecture on nanoscale and a 3D porous network structure on macroscale. ${ }^{[16]}$ Furthermore, recent studies suggest that multimetallic alloying offers new possibilities for boosting electrocatalysis performance. ${ }^{[26-29]}$ To this end, we chose the ethanol oxidation reaction (EOR) to evaluate the electrocatalytic properties of the multimetallic hierarchical aerogels. Before the EOR test, the aerogels were subjected to cyclic voltammograms (CVs) in $1 \mathrm{M} \mathrm{NaOH}$ solution to clean and activate the surface (Figure S12). The electrochemical active surface areas (ECSA) were estimated by employing the reduction peak of Pd/Pt oxide and assuming a charge density of $430 \mathrm{mC} \mathrm{cm}^{-2}$ for the formation of a monolayer Pd/Pt oxide. ${ }^{[30]}$ The Ni-Pd $\mathrm{Pt}_{\mathrm{y}}$ aerogels exhibit higher ECSA values (55.5, 54.7, 54.3, 52.3, 49.2, $46.9 \mathrm{~m}^{2} \mathrm{~g}^{-1}$ with the Pd:Pt ratio ranging from 1:0 to 0:1) than both the Pd/C and Pt/C catalysts (42.3 and $44.3 \mathrm{~m}^{2} \mathrm{~g}^{-1}$, respectively). The higher ECSA values indicate more accessible active sites, suggesting an increase in the utilization efficiency of noble metals. As depicted in Figure 5a, the polarization curves show considerably higher current and more negative onset potentials of ethanol oxidation 
for the $\mathrm{Ni}-\mathrm{Pd}_{\mathrm{x}} \mathrm{Pt}_{\mathrm{y}}$ aerogels than the $\mathrm{Pd} / \mathrm{C}$ and $\mathrm{Pt} / \mathrm{C}$, highlighting the importance of the multimetallic alloying effect in the enhancement of EOR kinetics. The mass activities (MAs) and specific activities (SAs) were evaluated from the CVs recorded at a sweep rate of $50 \mathrm{mV} \mathrm{s}^{-1}$ by normalizing with respect to the loading amount of noble metals and ECSA (Figure S13-14). As summarized in Figure $\mathbf{5 b}$, the MAs of the $\mathrm{Ni}^{-} \mathrm{Pd}_{\mathrm{x}} \mathrm{Pt}_{\mathrm{y}}$ aerogels are largely improved in comparison to the commercial $\mathrm{Pd} / \mathrm{C}$ and $\mathrm{Pt} / \mathrm{C}$. The Ni-Pd $60 \mathrm{Pt}_{40}$ aerogel shows the highest $\mathrm{MA}$ (6.87 $\mathrm{A} \mathrm{mg}^{-1}$ ) with an impressive improvement factor of 10.6 and 7.6 over the commercial $\mathrm{Pd} / \mathrm{C}$ and $\mathrm{Pt} / \mathrm{C}$, respectively (Figure 5c). The $\mathrm{Ni}-\mathrm{Pd}_{\mathrm{x}} \mathrm{Pt}_{\mathrm{y}}$ aerogels also display higher $\mathrm{SAs}$ and the $\mathrm{Ni}$ $\mathrm{Pd}_{60} \mathrm{Pt}_{40}$ aerogel exhibits the highest $\mathrm{SA}$ value $\left(12.7 \mathrm{~mA} \mathrm{~cm}{ }^{-2}\right)$, which is 8.5 and 6.4-fold higher than those of the $\mathrm{Pd} / \mathrm{C}$ and $\mathrm{Pt} / \mathrm{C}$ catalysts, respectively (Figure S14). In addition, the Ni-Pd${ }_{60} \mathrm{Pt}_{40}$ aerogel shows 1.5-fold and 1.3-fold improvement of the MA and SA, respectively, compared to the $\mathrm{Pd}_{60} \mathrm{Pt}_{40}$ aerogels (Figure S19).

The higher MA and SA of the ternary Ni-Pdx $\mathrm{Pt}_{\mathrm{y}}$ aerogels over the binary NiPd and NiPt aerogels indicates that the Pd-Pt complexing plays an important role in boosting their electrocatalytic activities. The contribution from the Ni doping was demonstrated by the higher activities of the $\mathrm{Ni}-\mathrm{Pd}_{\mathrm{x}} \mathrm{Pt}_{\mathrm{y}}$ aerogels over the Ni-free $\mathrm{Pd}_{\mathrm{x}} \mathrm{Pt}_{\mathrm{y}}$ aerogels. In addition, the hierarchical aerogel structures offer the advantages of high porosity, large surface area and synergistic interactions to the aerogel catalysts, which facilitate the mass transfer and expose more active sites for catalysis. Consequently, the improvement of the catalytic performance of the hierarchical Ni-Pdx $\mathrm{Pt}_{\mathrm{y}}$ aerogels is ascribed to two aspects: (i) multimetallic alloying effect, including the Pd-Pt complexing and the Ni doping; (ii) hierarchical structure, including the controlled morphology of the building blocks and the 3D porous aerogel structure. The synergy of the aforementioned effects contributes simultaneously to the large activity improvement of the multimetallic 
hierarchical aerogels, while the self-supportability guarantees high stability due to the elimination of carbon corrosion. ${ }^{[26]}$ The durability of the multimetallic aerogels was studied by chronoamperometry and CV cycling tests (Figure 5d and Figure S15-S18). The porous structure of the NBBs, the 3D aerogel backbone and the MA of the aerogel catalysts are mostly retained even after $2000 \mathrm{~s}$ of chronoamperometry test or 100 cycles of stability test.

In summary, we have demonstrated a new class of multimetallic hierarchical aerogels which are derived from alloyed $\mathrm{Ni}-\mathrm{Pd}_{\mathrm{x}} \mathrm{Pt}_{\mathrm{y}} \mathrm{NBBs}$ with in-situ engineered morphologies and compositions. The hierarchical aerogels combine two levels of porous structures, including the nanoscale hollow-spherical or dendritic morphologies of the NBBs and the macroscale aerogel nature, which integrates several catalysis enhancement factors such as high porosity, large surface area, multimetallic alloying, and self-supportability. The underlying mechanism of the galvanic shapeengineering is elucidated in terms of nanowelding of INPs based on time-dependent TEM studies and MD simulations. As a demonstration, the lower welding ability of Pt-dominant INPs leads to insufficient connection and welding of the INPs during the galvanic reaction, which results in a dendritic structure. This unconventional mechanism of galvanic replacement holds the potential for inspiring future work on galvanic synthesis. The EOR activity was largely improved upon the multimetallic hierarchical aerogels, and the $\mathrm{Ni}-\mathrm{Pd}_{60} \mathrm{Pt}_{40}$ aerogel exhibits the highest MA and SA values, which are 10.6 and 7.6-fold higher than those of the $\mathrm{Pd} / \mathrm{C}$ and $\mathrm{Pt} / \mathrm{C}$ catalysts. This work highlights the great potential of multimetallic hierarchical aerogels as highly efficient electrocatalysts through engineering the NBBs by nanotechnology. This approach may be further generalized for hierarchical aerogels with other morphologies and different catalysis reactions.

\section{Supporting Information}


Supporting Information is available online from the Wiley Online Library or from the author.

\section{Acknowledgements}

The authors thank Renate Schulze for the ICP-OES analysis, Susanne Goldberg for the SEM measurements, Christine Damm and Xue Zhao for the help with TEM and XPS measurements. We also thank Yizhong Lu for the meaningful discussion of the electrocatalysis part. Support by the Structural Characterization Facilities Rossendorf at IBC is gratefully acknowledged. Financial support by the DFG (ZuK support the best) and the ERC (AdG AEROCAT) is gratefully acknowledged.

Received: ((will be filled in by the editorial staff))

Revised: ((will be filled in by the editorial staff)) Published online: ((will be filled in by the editorial staff))

[1] M. V. Kovalenko, L. Manna, A. Cabot, Z. Hens, D. V. Talapin, C. R. Kagan, V. I. Klimov, A. L. Rogach, P. Reiss, D. J. Milliron, P. Guyot-Sionnnest, G. Konstantatos, W. J. Parak, T. Hyeon, B. A. Korgel, C. B. Murray, W. Heiss, ACS Nano 2015, 9, 1012.

[2] S. S. Kistler, Nature 1931, 127, 741.

[3] J. L. Mohanan, I. U. Arachchige, S. L. Brock, Science 2005, 307, 397.

[4] N. C. Bigall, A. K. Herrmann, M. Vogel, M. Rose, P. Simon, W. Carrillo-Cabrera, D.

Dorfs, S. Kaskel, N. Gaponik, A. Eychmüller, Angew. Chem. 2009, 121, 9911; Angew. Chem. Int. Ed. 2009, 48, 9731.

[5] I. U. Arachchige, S. L. Brock, Acc. Chem. Res. 2007, 40, 801.

[6] V. Sayevich, B. Cai, A. Benad, D. Haubold, L. Sonntag, N. Gaponik, V. Lesnyak, A. Eychmüller, Angew. Chem. 2016, 128, 6442; Angew. Chem. Int. Ed. 2016, 55, 6334.

[7] F. J. Heiligtag, W. Cheng, V. R. de Mendonca, M. J. Suess, K. Hametner, D. Gunther, C. Ribeiro, M. Niederberger, Chem. Mater. 2014, 26, 5576.

[8] H. Sun, Z. Xu, C. Gao, Adv. Mater. 2013, 25, 2554.

[9] Z. Xu, Y. Zhang, P. Li, C. Gao, ACS Nano 2012, 6, 7103.

[10] H. Yu, S. L. Brock, ACS Nano 2008, 2, 1563.

[11] W. Liu, A. K. Herrmann, N. C. Bigall, P. Rodriguez, D. Wen, M. Oezaslan, T. J. Schmidt, N. Gaponik, A. Eychmüller, Acc. Chem. Res. 2015, 48, 154.

[12] C. Zhu, Q. Shi, S. Fu, J. Song, H. Xia, D. Du, Y. Lin, Adv. Mater. 2016, 28, 8779.

[13] D. Wen, W. Liu, D. Haubold, C. Zhu, M. Oschatz, M. Holzschuh, A. Wolf, F. Simon, S. Kaskel, A. Eychmüller, ACS Nano 2016, 10, 2559.

[14] W. Liu, P. Rodriguez, L. Borchardt, A. Foelske, J. Yuan, A. K. Herrmann, D. Geiger, Z. Zheng, S. Kaskel, N. Gaponik, R. Kotz, T. J. Schmidt, A. Eychmüller, Angew. Chem. 2013, 125, 10033; Angew. Chem. Int. Ed. 2013, 52, 9849.

[15] D. Wen, A. K. Herrmann, L. Borchardt, F. Simon, W. Liu, S. Kaskel, A. Eychmüller, J. Am. Chem. Soc. 2014, 136, 2727.

[16] B. Cai, D. Wen, W. Liu, A. K. Herrmann, A. Benad, A. Eychmüller, Angew. Chem. 2015, 127, 13293; Angew. Chem. Int. Ed. 2015, 54, 13101.

[17] K. D. Gilroy, A. Ruditskiy, H. C. Peng, D. Qin, Y. Xia, Chem. Rev. 2016, 116, 10414.

[18] X. Xia, Y. Wang, A. Ruditskiy, Y. Xia, Adv. Mater. 2013, 25, 6313. 
[19] N. Gaponik, A.-K. Herrmann, A. Eychmüller, J. Phys. Chem. Lett. 2011, 3, 8.

[20] C. Zhu, D. Du, A. Eychmüller, Y. Lin, Chem. Rev. 2015, 115, 8896.

[21] Y. Wang, S. Zou, W.-B. Cai, Catalysts 2015, 5, 1507.

[22] D. C. Harris, L. J. Cabri, Can. Mineral. 1991, 29, 231.

[23] Y. G. Sun, Y. N. Xia, J. Am. Chem. Soc. 2004, 126, 3892.

[24] M. Grouchko, P. Roitman, X. Zhu, I. Popov, A. Kamyshny, H. Su, S. Magdassi, Nat. Comm. 2014, 5, 2994.

[25] D. V. Wagle, G. A. Baker, Mater. Horiz. 2015, 2, 157.

[26] A. Kowal, M. Li, M. Shao, K. Sasaki, M. B. Vukmirovic, J. Zhang, N. S. Marinkovic, P. Liu, A. I. Frenkel, R. R. Adzic, Nat. Mater. 2009, 8, 325.

[27] M. Li, D. A. Cullen, K. Sasaki, N. S. Marinkovic, K. More, R. R. Adzic, J. Am. Chem. Soc. 2013, 135, 132.

[28] Y. Zhang, Y.-C. Hsieh, V. Volkov, D. Su, W. An, R. Si, Y. Zhu, P. Liu, J. X. Wang, R. R. Adzic, ACS Catal. 2014, 4, 738.

[29] M. Li, P. Liu, R. R. Adzic, J. Phys. Chem. Lett. 2012, 3, 3480.

[30] Y. Lu, Y. Jiang, H. Wu, W. Chen, J. Phys. Chem. C 2013, 117, 2926. 

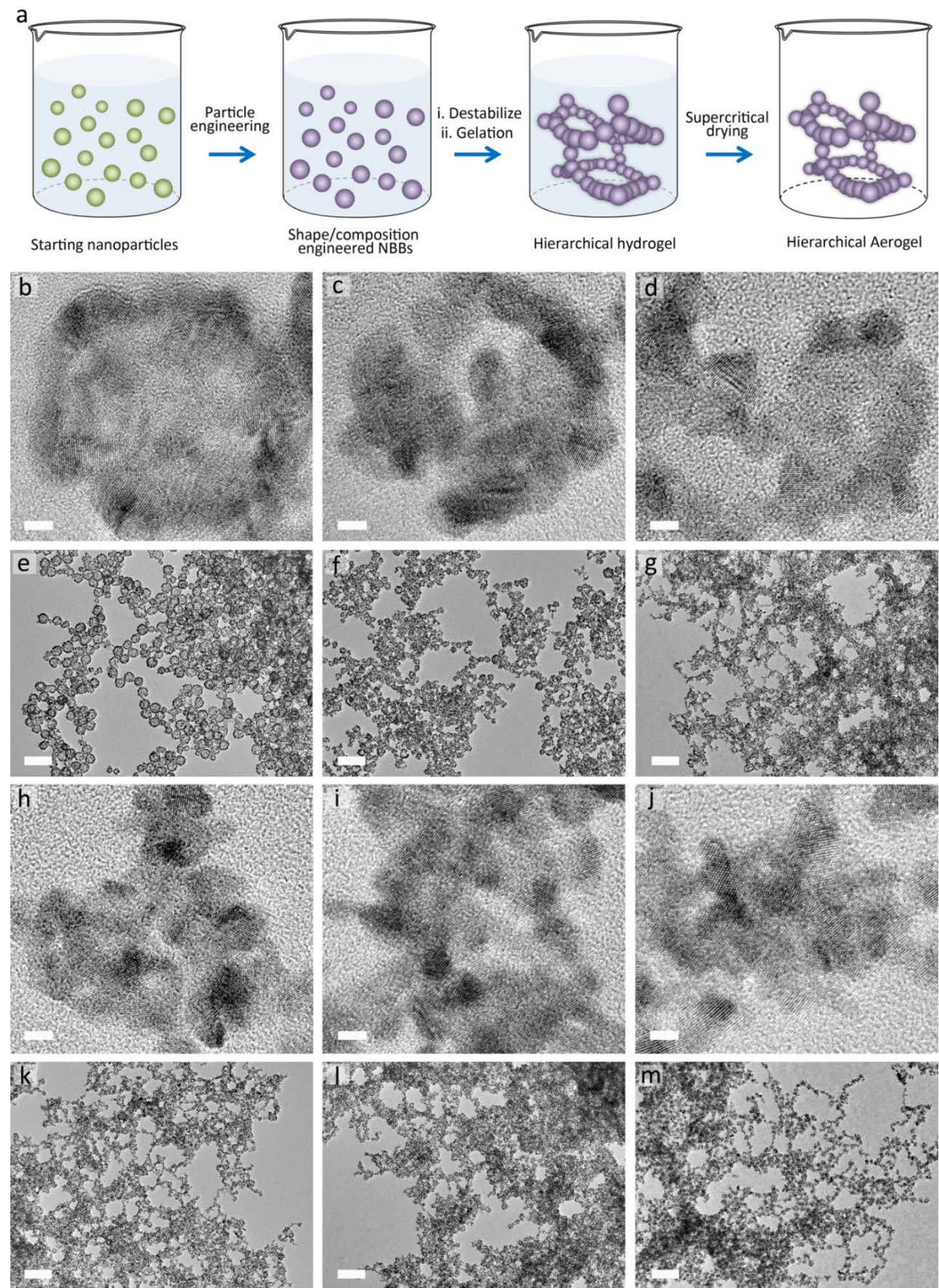

Figure 1. Shape and composition engineering of the NBBs for the hierarchical aerogels. a) Schematic illustration of the synthesis procedure. HR-TEM and low magnification images of the $\mathrm{Ni}-\mathrm{Pd}_{\mathrm{x}} \mathrm{Pt}_{\mathrm{y}}$ aerogels with different compositions: b, e) Ni-Pd, c, f) Ni-Pd ${ }_{80} \mathrm{Pt}_{20}$, d, g) Ni-Pd ${ }_{60} \mathrm{Pt}_{40}$, h, k) Ni-Pd $\left.{ }_{40} \mathrm{Pt}_{60}, \mathrm{i}, \mathrm{l}\right) \mathrm{Ni}^{-} \mathrm{Pd}_{20} \mathrm{Pt}_{80}, \mathrm{j}, \mathrm{m}$ ) Ni-Pt. Scale bars are $2 \mathrm{~nm}$ and $100 \mathrm{~nm}$ for HR-TEM and TEM images, respectively. 

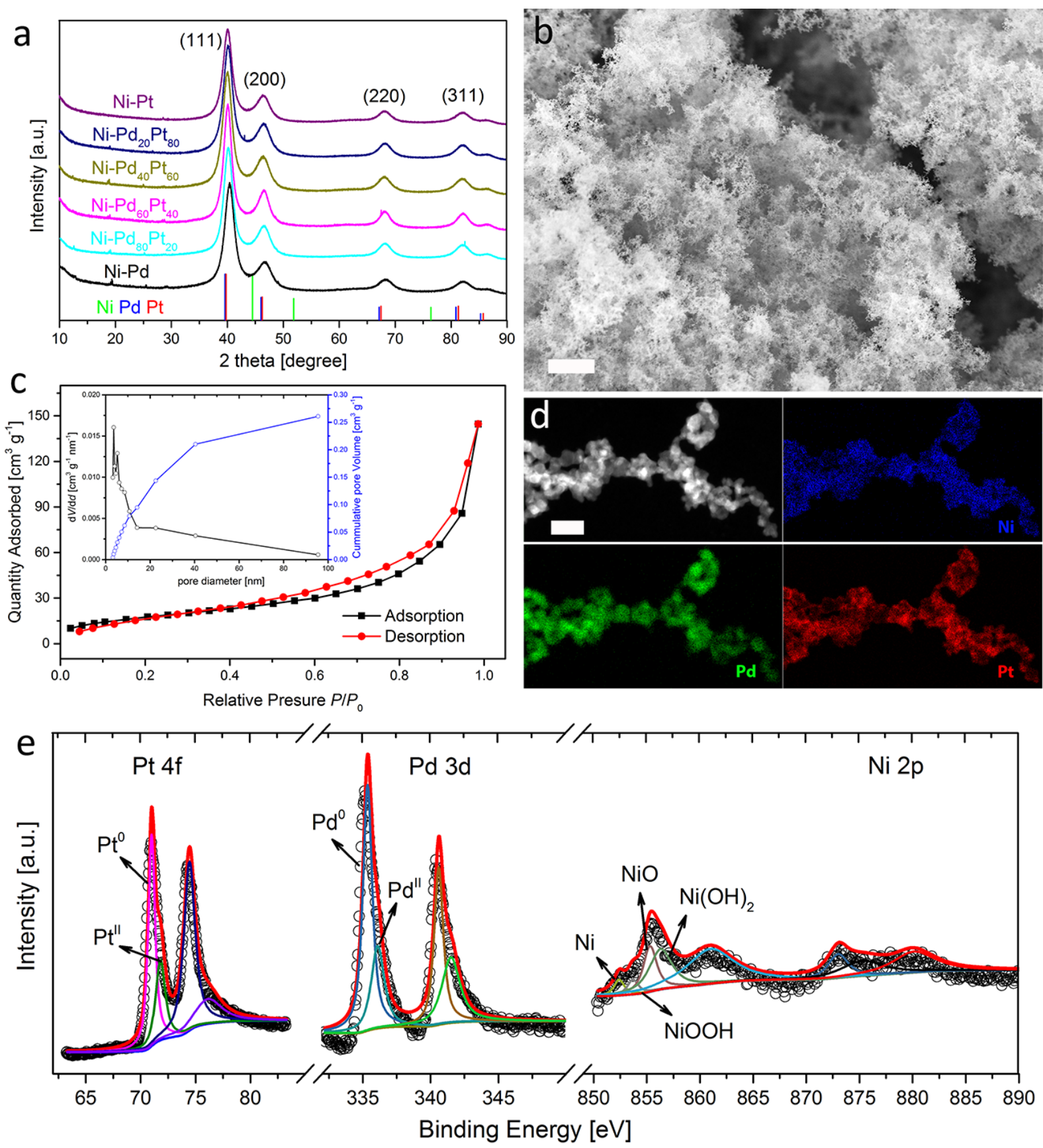

Figure 2. a) XRD analysis of the $\mathrm{Ni}-\mathrm{Pd}_{\mathrm{x}} \mathrm{Pt}_{\mathrm{y}}$ aerogels with different compositions. b) SEM image, c) $\mathrm{N}_{2}$ physisorption isotherms as well as pore size distribution and cumulative pore volumes (inset), d) high-angle annular dark-field (HAADF) STEM micrograph with corresponding element maps and e) XPS spectra of the aerogel taking the $\mathrm{Ni}-\mathrm{Pd}_{60} \mathrm{Pt}_{40}$ as example. Scale bars are b) $1 \mu \mathrm{m}$ and d) $10 \mathrm{~nm}$. 


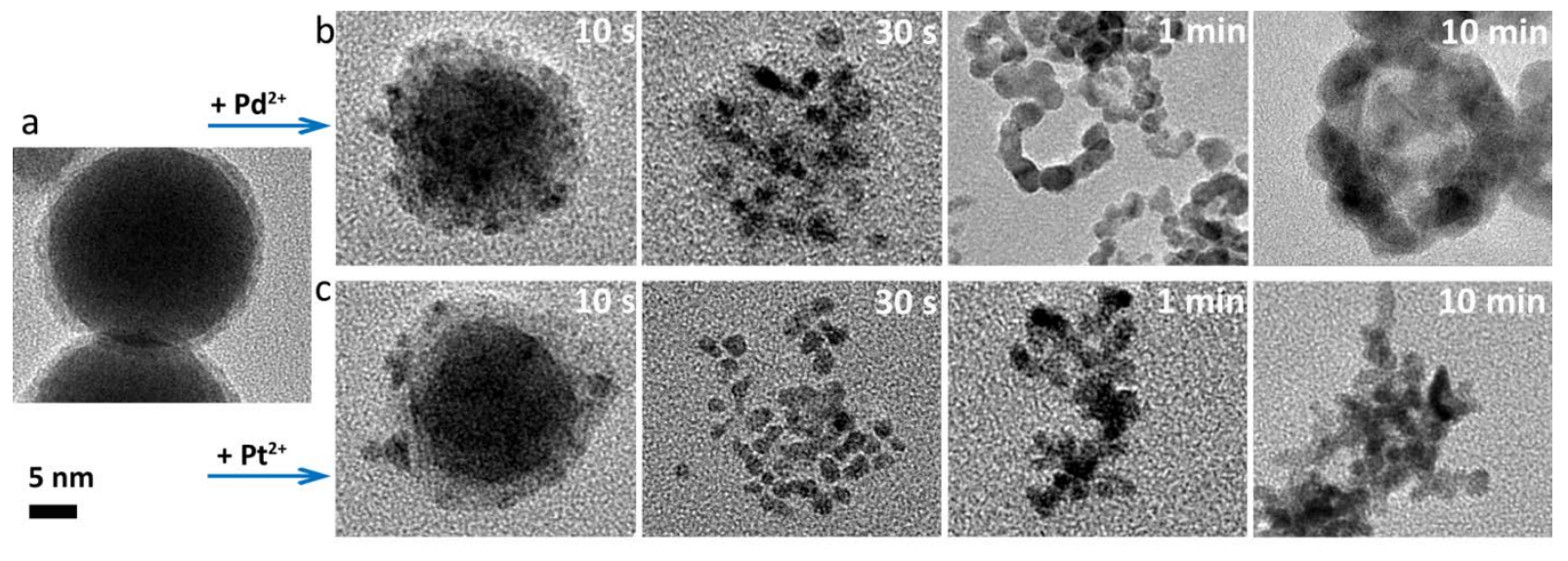

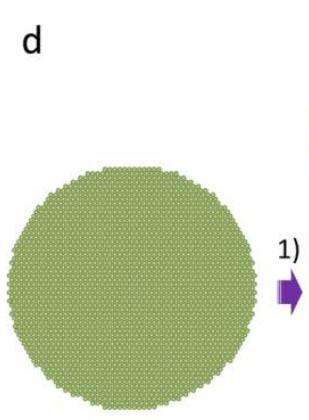

Ni NPs
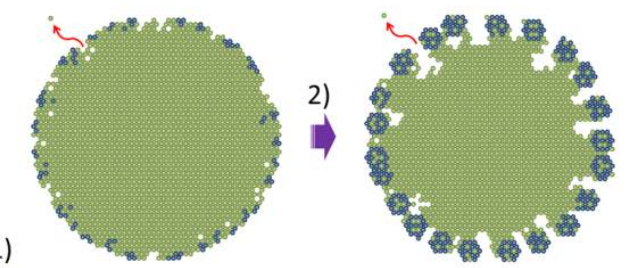

3)
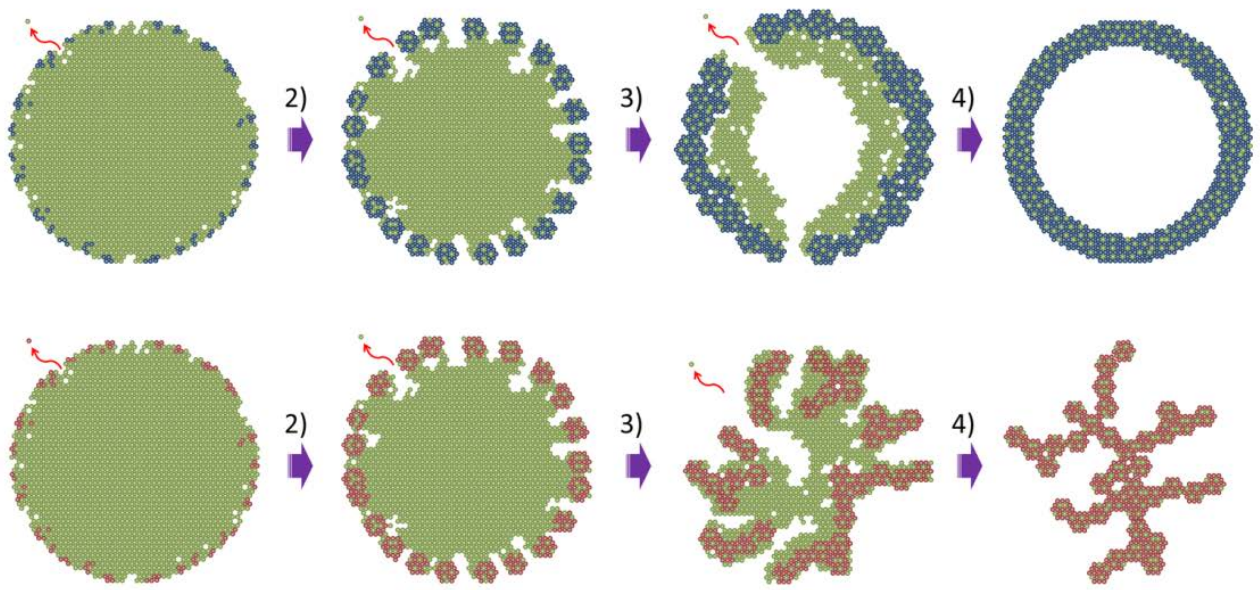

4)

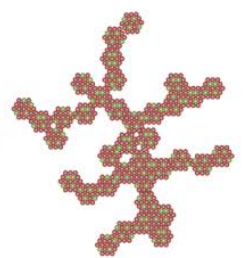

- Ni atom ०Pd atom $\circ \mathrm{Pt}$ atom

Figure 3. Structural growth mechanism of the edge composition systems. Time-dependent TEM images of a) Ni NPs and b) Ni-Pd and c) Ni-Pt systems monitored at different reaction times. d) Schematic illustration of the proposed growth mechanism of the Ni-Pd and Ni-Pt systems. It should be noted that the $\mathrm{NiO}_{\mathrm{x}}$ shell is unavoidable because of the ambient oxygen during the preparation of TEM grids. 

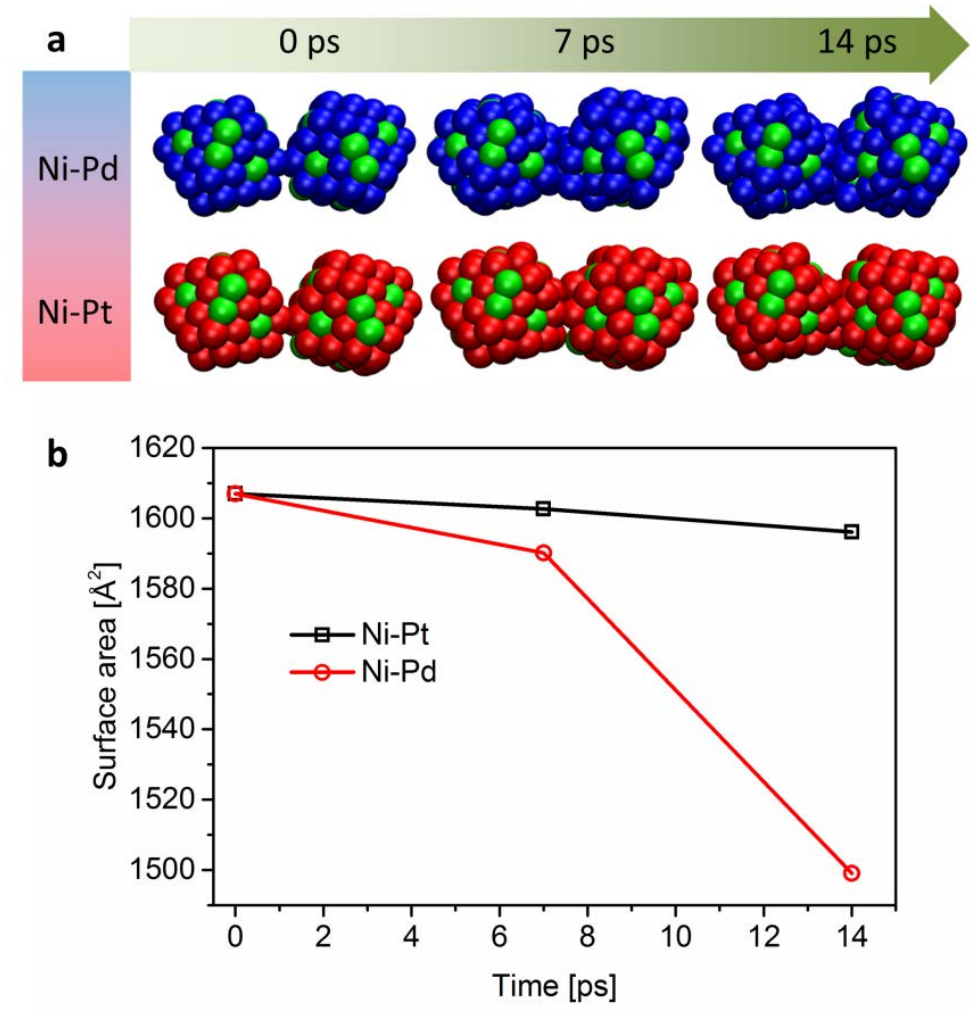

Figure 4. Molecular dynamics simulation of the nanowelding behavior of NiPd and NiPt particle systems. a) Time evolution of the welding process of $\mathrm{Ni}_{20} \mathrm{Pd}_{80}$ and $\mathrm{Ni}_{20} \mathrm{Pt}_{80}$ systems comprising 2 particles adjacent to (111) facets. b) Comparison of the welding rate of the $\mathrm{Ni}_{20} \mathrm{Pd}_{80}$ and $\mathrm{Ni}_{20} \mathrm{Pt}_{80}$ systems based on total surface area. 

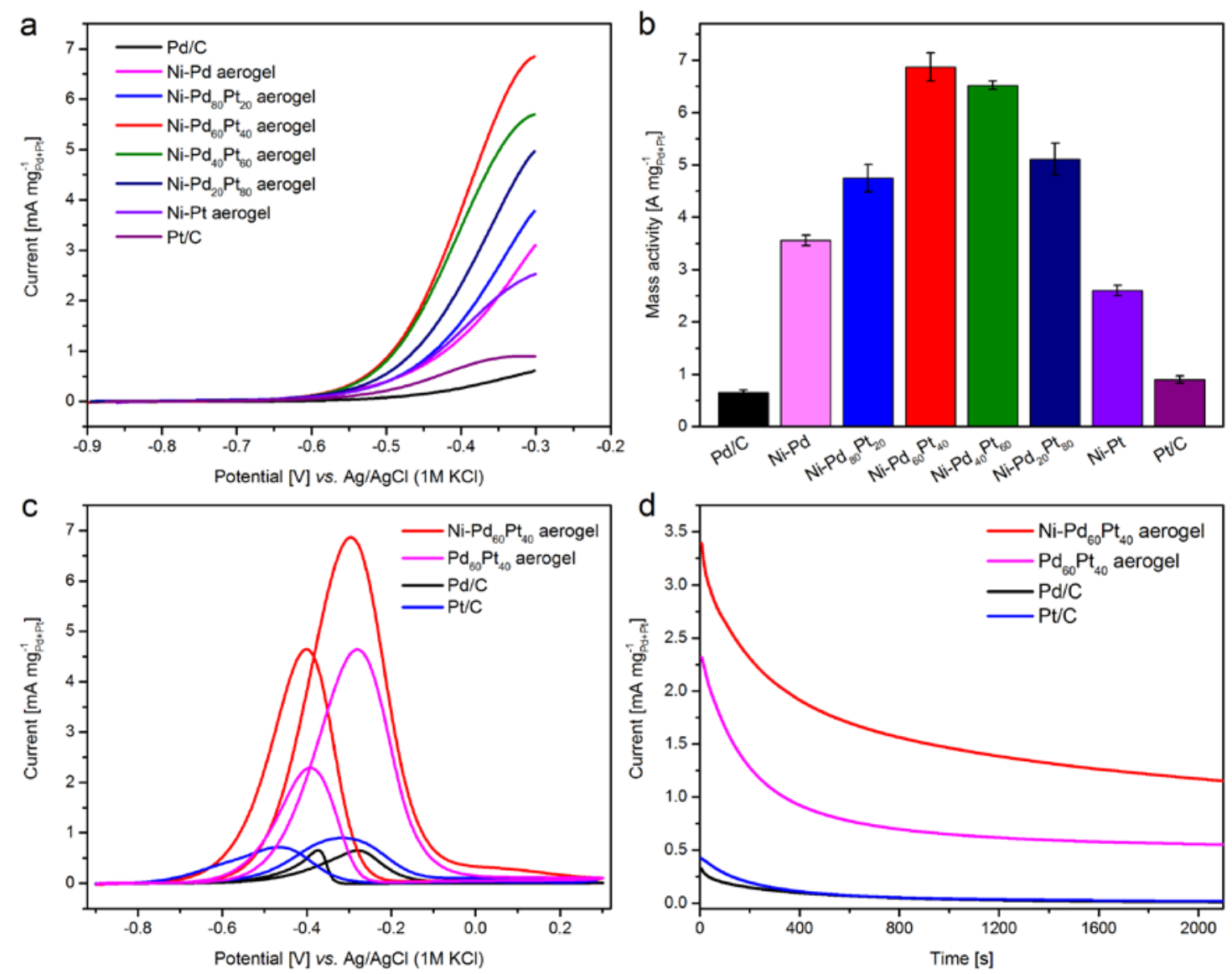

Figure 5. Electrocatalytic performance. a) Noble-metal-mass normalized polarization curves of EOR and b) mass activities of the Ni-Pd $\mathrm{Pt}_{\mathrm{y}}$ aerogels with different compositions and the $\mathrm{Pd} / \mathrm{C}$ and $\mathrm{Pt} / \mathrm{C}$ catalysts. c) Noble-metal-mass normalized CVs and d) i-t curves of the Ni-Pd${ }_{60} \mathrm{Pt}_{40}$ hierarchical aerogel in comparison with the $\mathrm{Pd}_{60} \mathrm{Pt}_{40}$ aerogel, $\mathrm{Pd} / \mathrm{C}$ and $\mathrm{Pt} / \mathrm{C}$ catalysts. The loading of noble metal is $20 \mu \mathrm{g} \mathrm{cm}^{-2}$. Scan rate: $50 \mathrm{mV} \mathrm{s}^{-1}$. Solution for EOR: $1 \mathrm{M} \mathrm{NaOH}+1 \mathrm{M}$ ethanol. 


\section{Table of contents}

A new class of multimetallic hierarchical aerogels composed entirely of interconnected $\mathrm{Ni}$ $\mathrm{Pd}_{\mathrm{x}} \mathrm{Pt}_{\mathrm{y}}$ nano building blocks with in-situ engineered morphologies and compositions is demonstrated. The underlying mechanism of the galvanic shape-engineering is elucidated in terms of nanowelding of intermediate nanoparticles. The hierarchical aerogels integrate two levels of porous structures, leading to improved electrocatalysis performance.

Keyword aerogels, hierarchical structures, porous metals, sol-gel processes, electrocatalysts

Bin Cai, Arezoo Dianat, René Hübner, Wei Liu, Dan Wen, Albrecht Benad, Luisa Sonntag, Gianaurelio Cuniberti and Alexander Eychmüller*

Multimetallic Hierarchical Aerogels: Shape-engineering of the Building Blocks for efficient electrocatalysis

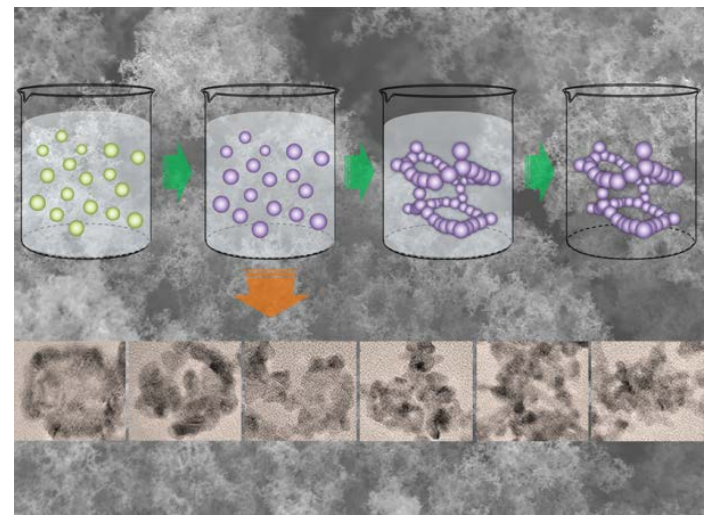

